\title{
Fingerprinting methicillin-resistant Staphylococcus aureus by the immunoblot technique
}

\author{
WOEI LEE and J. P. BURNIE* \\ Department of Medical Microbiology, St Bartholomew's Hospital, West Smithfield, London EC1A 7BE
}

\begin{abstract}
Summary. A series of 133 isolates of methicillin-resistant Staphylococcus aureus was fingerprinted by the immunoblot technique. Extracts were prepared by lysostaphin degradation of overnight cultures and peptides were separated by SDS polyacrylamide gel electrophoresis. The peptides were transblotted on to nitrocellulose membranes and probed with (1) a hyperimmune rabbit serum raised against a methicillinresistant $S$. aureus isolate, (2) a hyperimmune rabbit serum raised against an isolate of $S$. epidermidis, and (3) serum from a patient who had recovered from an infection with a methicillin-resistant $S$. aureus. This typing method confirmed the existence of an epidemic strain that accounted for 102 of the isolates. The remaining 31 isolates were grouped into a further seven types which correlated with the results of phage typing and antibiograms.
\end{abstract}

\section{Introduction}

Recently there has been an increase in interest in methicillin-resistant Staphylococcus aureus (MRSA) as a cause of serious nosocomial infection in hospitals. Some affected hospitals report that MRSA may account for $20-40 \%$ of all clinical isolates of $S$. aureus (Combined working party, 1986). Resistance to methicillin means that treatment with flucloxacillin and the other isoxazolyl penicillins is contraindicated (Sanderson, 1986). These isolates are usually resistant also to penicillin, gentamicin, cephalosporins, erythromycin and tetracyclines. Therefore, the mainstay of treatment is vancomycin (McDonald et al., 1981; Marples et al., 1986). This antibiotic is toxic and needs regular monitoring; to avoid its unneccessary use, the correct identification of methicillin-resistant isolates is important.

There has been much debate about the pathogenicity of MRSA strains and it has been suggested that the genetic material required for multiple antibiotic resistance might limit their ability to spread from patient to patient (Lacey and Chopra, 1975). More recent epidemiological data have suggested that there are strains of MRSA that are as virulent as methicillin-sensitive strains (Crossley et al., 1979; Boyce et al., 1981; Peacock et al., 1981) whereas others are less virulent (Lacey et al., 1986).

Received 9 April 1987; accepted 1 July 1987.

*Correspondence and requests for offprints should be sent to Dr Burnie.
The results of phage typing and antibiograms have confirmed that there is more than one type of MRSA and have demonstrated a predominant outbreak strain. This strain is recognised by its typing weakly with phage 85 , occasionally strongly with phage 84 , and strongly with experimental phages 88A and 932 (Marples et al., 1986). Isolates of this type have caused major outbreaks (Hone $e t$ al., 1981; Bradley et al., 1985; Shanson et al., 1985) the control of which is expensive and time consuming. Clearly it would be advantageous to be able to differentiate between isolates of the epidemic MRSA (EMRSA) type and other methicillinresistant staphylococci without recourse to the reference laboratory. This would confirm the results of antibiotic testing and allow the rapid instigation of control measures.

The examination of the sodium dodecyl sulphatepolyacrylamide gel electrophoresis (SDS-PAGE) profiles of different staphylococcal species has shown that each species produces a pattern distinct for that species. However, the patterns could not be used for typing isolates within species (Clink and Pennington, 1987). SDS-PAGE typing with $\left[{ }^{35} \mathrm{~S}\right]$-methionine has been valuable in fingerprinting isolates but this is an expensive technique that requires the use of radiolabels and it has been applied to only a few isolates (Stephenson et al., 1986). Isolates of methicillin-sensitive $S$. aureus have been typed by immunoblotting culture supernate against human antiserum (Krikler et al., 1986). The results agreed with those found by phage 
typing but the technique was not extended to methicillin-resistant strains. Isolates were grouped after visual inspection of immunoblots on the basis of the possession of bands unique to a group, band intensity and the presence or absence of bands common to several groups. A single antibody probe was used.

We developed a similar immunoblot fingerprinting system for Candida albicans (Lee et al., 1986) and demonstrated that the enzymic breakdown of whole cells produced a better antigenic preparation in terms of the amount of protein available for typing and reproducibility. Isolates identical on fingerprinting with one antibody probe remained indistinguishable with a second probe although the overall pattern might have been quite different (Burnie and Matthews, 1987). It was shown that a difference in three antigenic bands, as defined by the presence or absence of a particular band or a very great variation in its density, was necessary to prove that two isolates were definitely different. Discrimination could be improved by examining the results obtained with multiple antibody probes (Lee et al., 1986).

This paper presents data on 133 methicillinresistant isolates of $S$. aureus immunoblotted against three antibody probes. Lysostaphin-degraded whole-cell extracts of all the isolates were tested against a rabbit hyperimmune antiserum raised against a French-press extract of an EMRSA strain. Examples of the different types generated were then fingerprinted with a serum from a patient who had recovered from a systemic infection with the EMRSA and against a rabbit hyperimmune antiserum raised against a French-press extract of an isolate of $S$. epidermidis.

\section{Materials and methods}

\section{Isolates}

The test series of 133 isolates was obtained from the London Hospital (87), the London Chest Hospital (3), St Bartholomew's Hospital (10), Reading (1), Oldchurch (2), Hull Royal Infirmary (8), St James' Hospital, Leeds (2), Pinderfields Hospital, Wakefield (6), St Luke's Hospital (9), Australia (4) and Egypt (1). They were all slide- and tube-coagulase positive.

\section{Antibiograms}

These were determined by a disk susceptibility method on Isosensitest Agar (Oxoid CM 471) with 10- $\mu$ g disks of methicillin, tetracycline, gentamicin, erythromycin, neomycin, fusidic acid and chloramphenicol, 2- $\mu \mathrm{g}$ disks of rifampicin and clindamycin and $1-\mu \mathrm{g}$ disk of penicillin.
All tests were performed at $35^{\circ} \mathrm{C}$ with the exception of that with methicillin which was performed at $30^{\circ} \mathrm{C}$.

\section{Phage typing}

This was performed by the Division of Hospital Infection, Colindale by the standard method (Parker, 1972).

\section{Immunoblotting}

Preparation of antigen. Isolates were grown overnight in $2 \mathrm{ml}$ of Tryptone Soya Broth at $37^{\circ} \mathrm{C}$. Cells were harvested by centrifugation at $3000 \mathrm{~g}$ for $10 \mathrm{~min}$. The pellet was resuspended in $100 \mu \mathrm{l}$ of sterile distilled water with $100 \mu \mathrm{l}$ of lysostaphin (Sigma) solution, initial concentration $200 \mu \mathrm{g} / \mathrm{ml}$, and incubated for $30 \mathrm{~min}$ on a shaking tray at $37^{\circ} \mathrm{C} ; 60 \mu \mathrm{l}$ of the resulting extract was loaded into each well.

Preparation of antiserum (i) Rabbit. An isolate of the EMRSA, as defined by antibiograms and phage typing (Marples et al., 1986), was grown overnight at $37^{\circ} \mathrm{C}$ on Blood Agar (Oxoid). Growth was harvested, centrifuged at $3000 \mathrm{~g}$ for $10 \mathrm{~min}$, resuspended and washed three times, all in distilled water. The washed cells were then treated in a French press at $12 \mathrm{~m}$ Pascals twice, and centrifuged at $12000 \mathrm{~g}$ for $10 \mathrm{~min}$. New Zealand White rabbits were inoculated with the resulting supernate; $25 \mathrm{mg}$ was mixed with $1 \mathrm{ml}$ of sterile water and $1 \mathrm{ml}$ of Freund's complete adjuvant. Animals were inoculated twice, with 14 days between, and bled after 28 days. The hyperimmune rabbit antiserum against the $S$. epidermidis isolate was prepared similarly, as described by Burnie and Matthews (1987).

(ii) Human. Several samples of human serum were obtained from a patient with an abdominal aortic graft infected with the epidemic strain of MRSA and pooled. This serum was shown by immunoblotting to be hyperimmune to the EMRSA.

Gel electrophoresis. The enzyme extracts were solubilised in sodium dodecyl sulphate (SDS) $2.6 \%$ and 2mercapto-ethanol $1.3 \%$ at $100^{\circ} \mathrm{C}$ for $5 \mathrm{~min}$. Electrophoresis of these preparations was performed with $10 \%$ polyacrylamide gels in a discontinuous buffer system (Laemmli, 1970). The following mol. wt $\left(10^{3}\right)$ standards were applied : myosin $200 ; \beta$-galactosidase 116.25 ; phosphorylase $B, 92.5$; bovine serum albumin $66 \cdot 2$; ovalbumin 45 ; carbonic anhydrase 31 ; soybean trypsin inhibitor $21 \cdot 5$; and lysozyme $14 \cdot 4$.

Immunoblots. Proteins were blotted on to nitrocellulose paper (Bio-Rad Laboratories, Richmond, CA, USA) in $25 \mathrm{~mm}$ Tris, $192 \mathrm{mM}$ glycine buffer, $p \mathrm{H} 8 \cdot 3$, containing methanol $20 \%$. Transfer was performed at $25^{\circ} \mathrm{C}$ with a current of $0.5 \mathrm{~A}$ for $45 \mathrm{~min}$ in an LKB Trans-Blot cell. Free protein sites were saturated by incubation in bovine serum albumin (Sigma) 3\% in buffered saline $(\mathrm{NaCl} 0.9 \%$ and $10 \mathrm{~mm}$ Tris, $p \mathrm{H} \mathrm{7.4)}$ at $4^{\circ} \mathrm{C}$ overnight. The nitrocellulose membrane was then incubated at $25^{\circ} \mathrm{C}$ for $2 \mathrm{~h}$ with either the rabbit antiserum (diluted 1 in 50) or the human serum (diluted 1 in 10 ) in bovine serum albumin $3 \%$ and 
Tween $200.05 \%$. After washing five times in saline $0.9 \%$ and Tween $200.05 \%$, the nitrocellulose was incubated for $1 \mathrm{~h}$ at $25^{\circ} \mathrm{C}$ with alkaline phosphatase-conjugated goat anti-rabbit IgG (Sigma) or, if a human antibody was the probe, a mixture of anti-human-IgG and anti-humanIgM immunoglobulin (Sigma), used at a 1 in 1000 dilution in bovine serum albumin 3\%. After washing again, the membrane was incubated for $15 \mathrm{~min}$ with freshly prepared mixtures of equal volumes of naphthol ASMX phosphate (Sigma; $0.4 \mathrm{mg} / \mathrm{ml}$ in distilled water) and fast red TR salt (Sigma; $6 \mu \mathrm{g} / \mathrm{ml}$ in 0.2 $\mathrm{M}$ Tris, $p \mathrm{H} 8 \cdot 2$ ).

\section{Results}

Immunoblotting detected multiple antigenic bands with mol. wts $\left(10^{3}\right) 38-175 ; 15$ of these were selected for the development of the fingerprinting system on the basis of their reproducibility between immunoblots. Probing with the rabbit hyperimmune anti-MRSA antibody confirmed the homogeneity of the EMRSA isolates. Immunoblots of extracts of six EMRSA isolates are illustrated in fig. 1, all from patients at the London Hospital. Tracks 2 and 3 were sequential isolates from the same patient. When the whole series of 133 isolates was examined eight different types were delineated

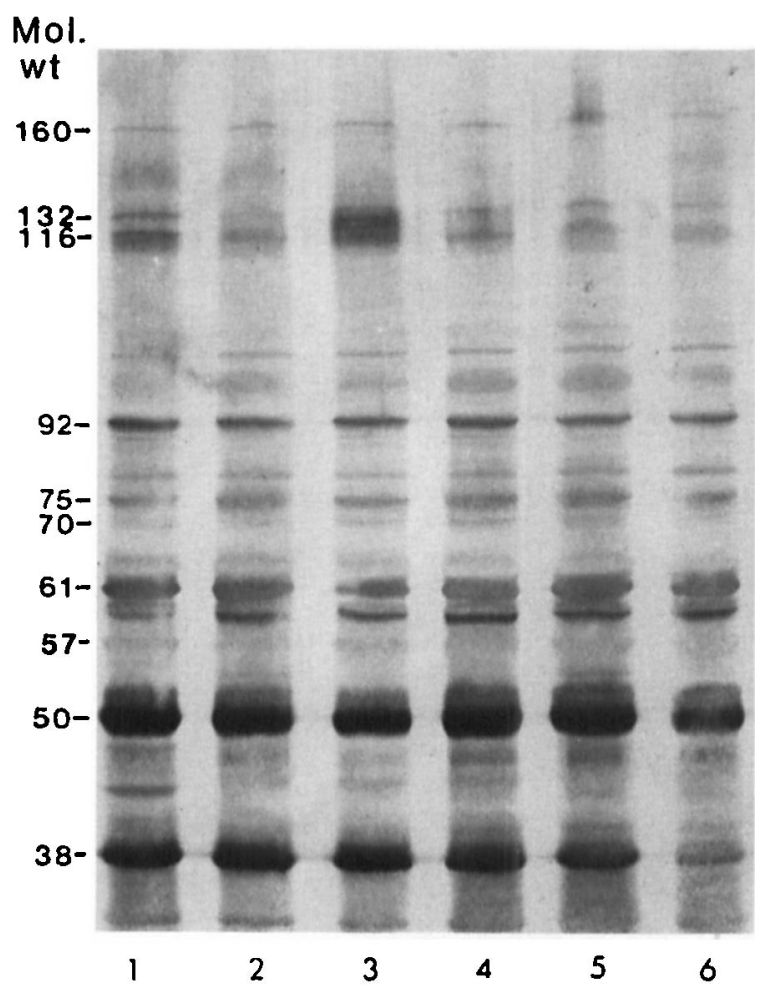

Fig. 1. Six isolates of the EMRSA (type 2) fingerprinted by the rabbit anti-EMRSA hyperimmune serum.
Table I. Results of immunoblot fingerprinting MRSAs

\begin{tabular}{|c|c|c|}
\hline Type & $\begin{array}{l}\text { Number of } \\
\text { isolates }\end{array}$ & Comments \\
\hline 1 & 3 & $\begin{array}{l}\text { Rifampicin resistant variant of the } \\
\text { EMRSA }\end{array}$ \\
\hline 2 & $102^{*}$ & EMRSA \\
\hline 3 & 1 & $\begin{array}{l}\text { Indistinguishable from EMRSA except } \\
\text { typed with phage } 83 \mathrm{~A}\end{array}$ \\
\hline 4 & 6 & $\begin{array}{l}\text { All from Pinderfields Hospital. Not } \\
\text { EMRSA }\end{array}$ \\
\hline 5 & 1 & Penicillin sensitive. Not EMRSA \\
\hline 6 & 16 & $\begin{array}{l}\text { All from Hull Royal Infirmary, St. James' } \\
\text { Hospital or St. Luke's Hospital. Not } \\
\text { EMRSA }\end{array}$ \\
\hline 7 & 3 & $\begin{array}{l}\text { Restricted to Hull Royal Infirmary. Not } \\
\text { EMRSA }\end{array}$ \\
\hline 8 & 1 & $\begin{array}{l}\text { Resistant to all antibiotics tested except } \\
\text { vancomycin; non-typable }\end{array}$ \\
\hline
\end{tabular}

* 102 EMRSA isolates from the London Hospital (85), St Bartholomew's Hospital (10), Reading (1), Australia (4) and Oldchurch (2).

(table I). These produced identical patterns when stained by Coomassie blue (fig. 2) but different patterns when transferred on to nitrocellulose membrane and probed by the rabbit anti-MRSA serum (fig. 3), rabbit anti-S. epidermidis serum (fig. 4) and the human serum (fig. 5). Antibody binding to a particular antigenic band was categorised as absent, trace or strong (tables II-IV).

Fingerprinting systems can be assesed by three standards: typability, reproducibility and discrimination. All isolates of methicillin-resistant $S$. aureus were typable by this method. Reproducibility between immunoblots was assessed by repeating tests with each isolate at least three times on different gels. Previous immunoblot fingerprinting methods have advocated the sequential analysis of isolates, one representative from each type being run on each subsequent gel to avoid the necessity of comparision between gels. This system was necessary for the fingerprinting of $C$. albicans (Lee et al., 1986) but the results for staphylococci were much more reproducible so that direct comparison between gels was possible. Track 2 in both figs 1 and 3 represents isolates of the EMRSA fingerprinted by the rabbit hyperimmune antiserum. The same pattern of antigenic bands was detected in both extracts although there was some difference in the intensity of the bands at mol. wts $\left(10^{3}\right) 160,132$, 116,75 and 57 because of the use of a different batch of rabbit antiserum.

The third criterion is discrimination. When isolates were typed by the rabbit hyperimmune 


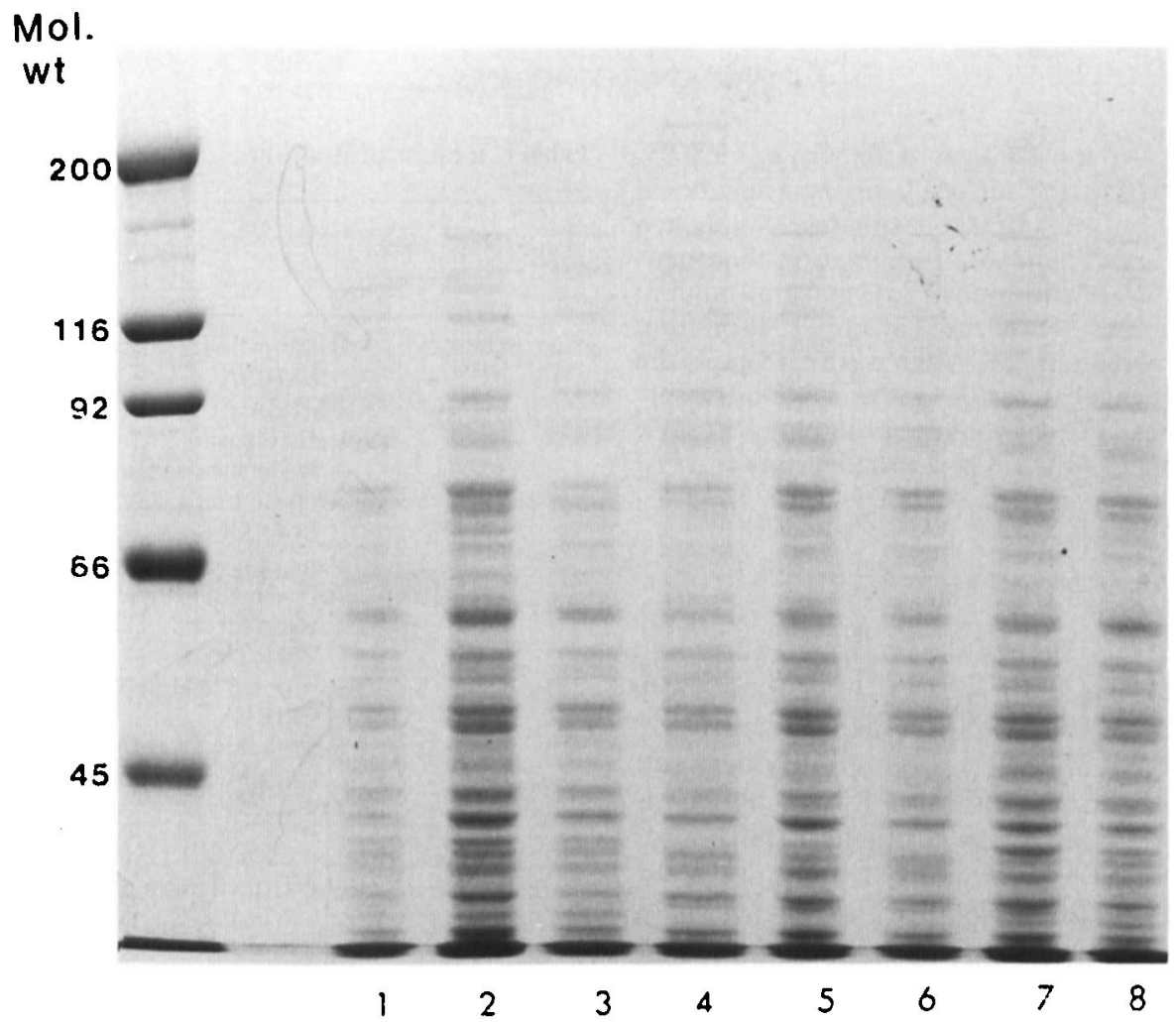

Fig. 2. Coomassie blue-stained extracts representing the eight types. The numbers refer to the type number given in table I. Mol. wts $\left(10^{3}\right)$ are represented by the markers in the left hand lane.

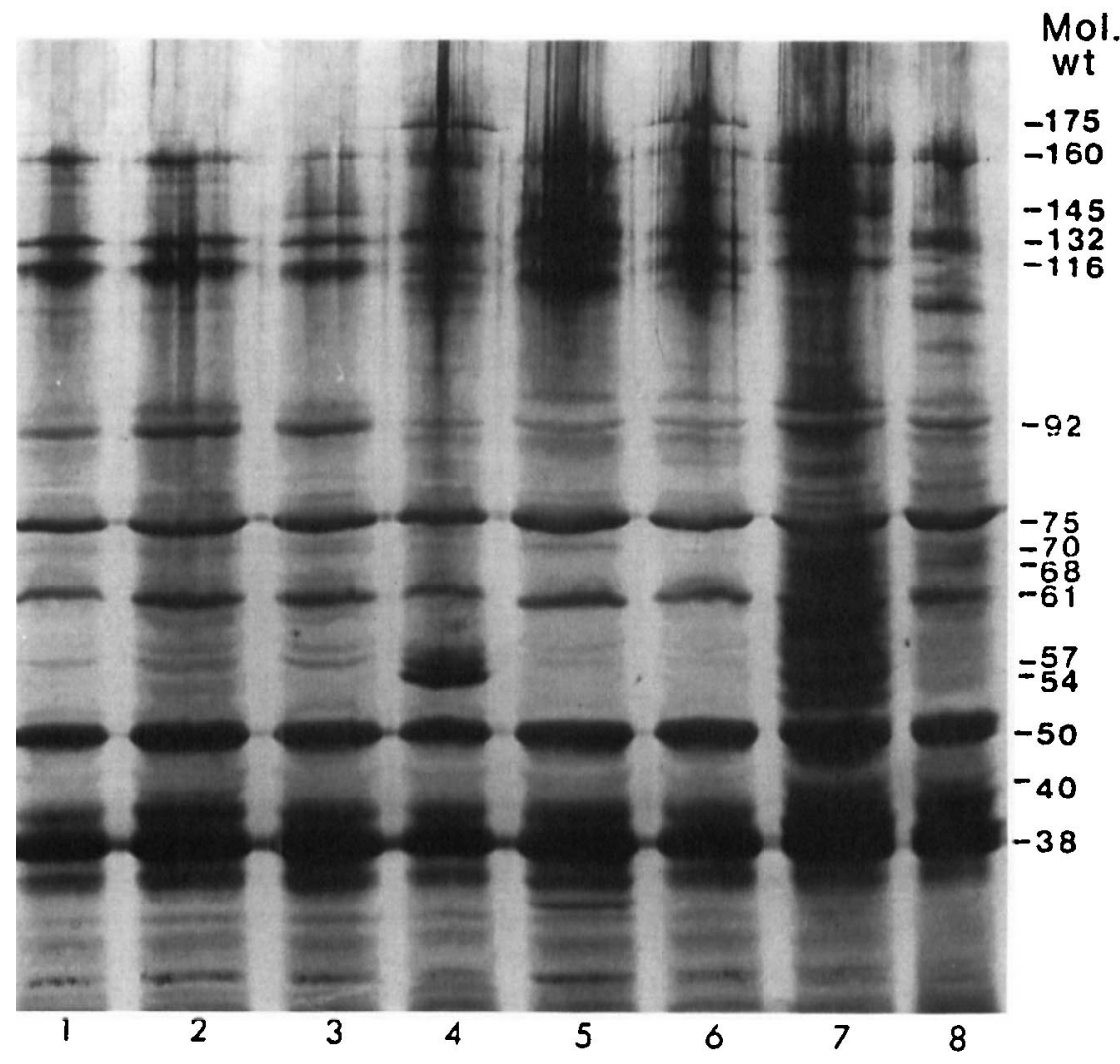

Fig. 3. Immunoblot of the eight types stained by the rabbit anti-EMRSA hyperimmune serum. The numbers refer to the type numbers given in table $\mathrm{I}$. Mol. wt $\left(10^{3}\right)$ are represented by the markers in the right hand lane. 


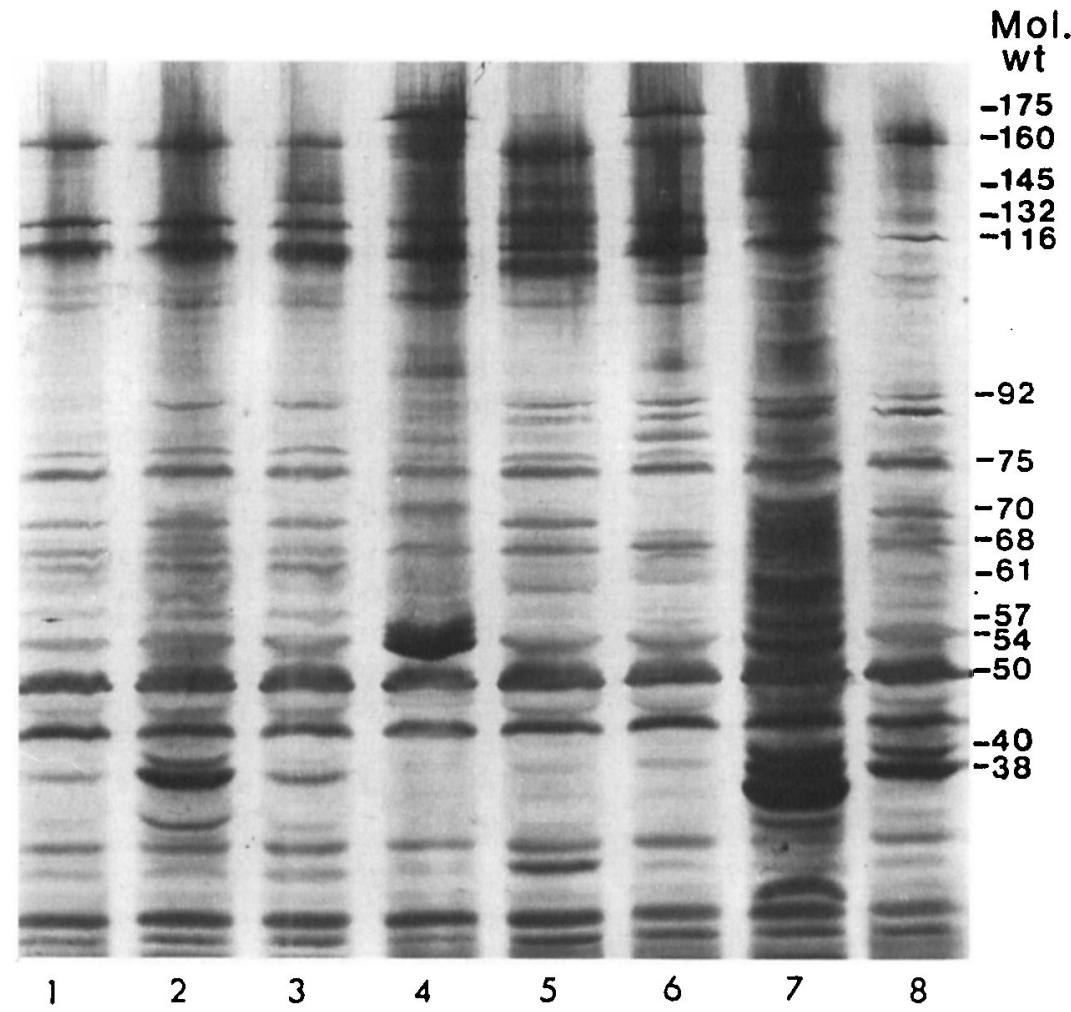

Fig. 4. Immunoblot of the eight types stained by the rabbit anti-S. epidermidis hyperimmune serum. The numbers refer to the type numbers given in table $\mathrm{I}$.

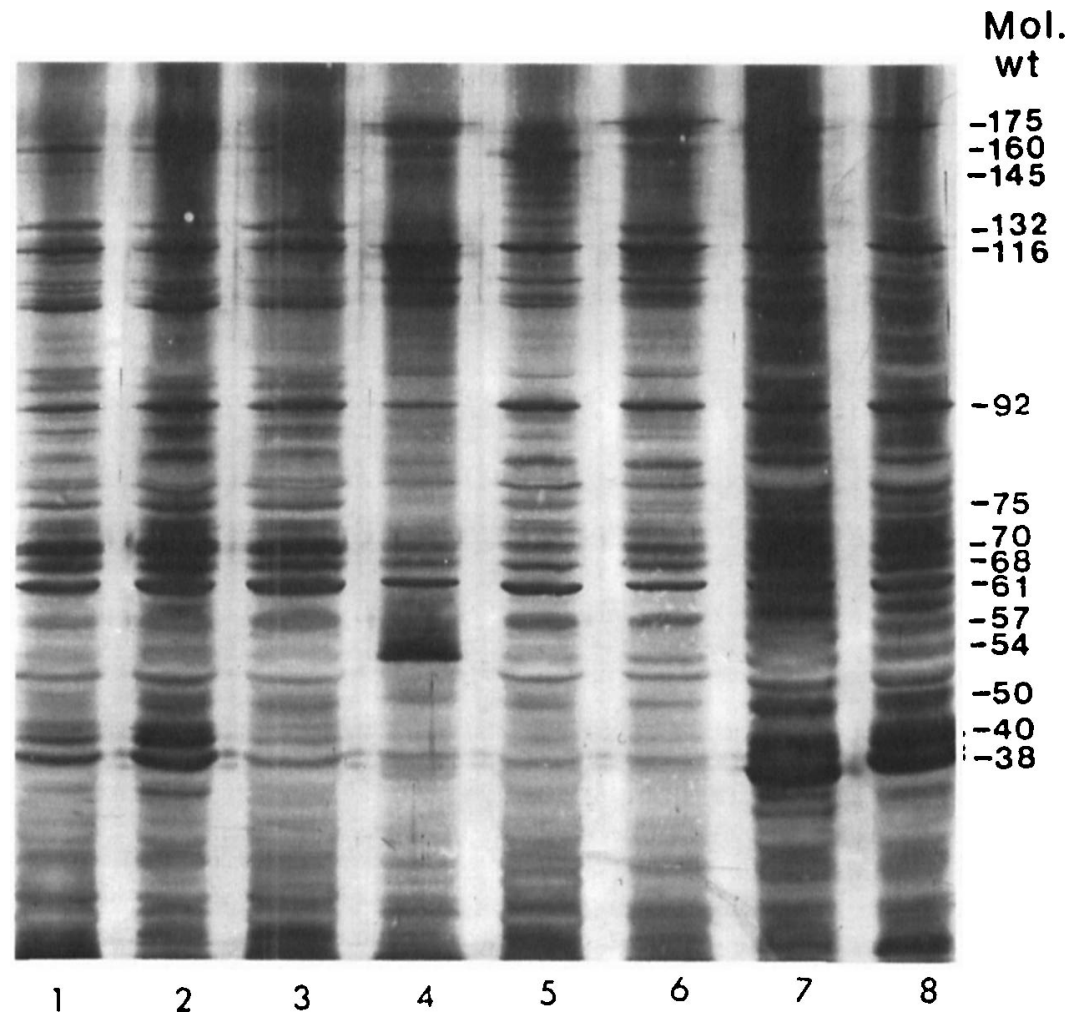

Fig. 5. Immunoblot of the eight types stained by human serum. The numbers refer to the type numbers given in table I. 
Table II. Results of the MRSA rabbit antiserum fingerprinting of the eight type strains

\begin{tabular}{|c|c|c|c|c|c|c|c|c|}
\hline \multirow{2}{*}{$\begin{array}{l}\text { Band } \\
\text { Mol. wt } \\
\left(10^{3}\right)\end{array}$} & \multicolumn{8}{|c|}{ Presence of the band in type } \\
\hline & 1 & 2 & 3 & 4 & 5 & 6 & 7 & 8 \\
\hline 38 & + & + & + & + & + & + & + & + \\
\hline 40 & - & - & - & - & - & - & + & + \\
\hline 50 & + & + & + & + & + & + & + & + \\
\hline 54 & - & - & - & + & - & - & + & - \\
\hline 57 & $\operatorname{tr}$ & $\operatorname{tr}$ & $\operatorname{tr}$ & + & - & - & + & - \\
\hline 61 & + & + & + & + & + & + & + & + \\
\hline 68 & - & - & - & - & - & - & + & + \\
\hline 70 & $\mathrm{tr}$ & $\mathrm{tr}$ & tr & - & $\mathrm{tr}$ & - & + & + \\
\hline 75 & + & + & + & + & + & + & + & + \\
\hline 92 & + & + & + & $\mathrm{tr}$ & $\mathrm{tr}$ & $\mathrm{tr}$ & + & + \\
\hline 116 & + & + & + & $\mathrm{tr}$ & - & $\mathrm{tr}$ & + & - \\
\hline 132 & + & + & + & + & + & + & - & + \\
\hline 145 & - & - & + & - & - & - & + & - \\
\hline 160 & + & + & + & + & + & + & + & + \\
\hline 175 & - & - & - & + & - & + & - & - \\
\hline
\end{tabular}

$-=$ Absent $;$ tr $=$ trace $;+=$ strong.

Table III. Results of the $S$. epidermidis rabbit antiserum fingerprinting of the eight type strains

\begin{tabular}{|c|c|c|c|c|c|c|c|c|}
\hline \multirow{2}{*}{$\begin{array}{l}\text { Band } \\
\text { Mol. wt } \\
\left(10^{3}\right)\end{array}$} & \multicolumn{8}{|c|}{ Presence of the band in type } \\
\hline & 1 & 2 & 3 & 4 & 5 & 6 & 7 & 8 \\
\hline 38 & $\operatorname{tr}$ & + & $\operatorname{tr}$ & - & - & - & + & + \\
\hline 40 & - & + & - & - & - & - & + & + \\
\hline 50 & + & + & + & + & + & + & + & + \\
\hline 54 & + & + & + & + & + & + & + & + \\
\hline 57 & - & - & - & + & - & - & + & - \\
\hline 61 & - & - & - & - & - & - & + & + \\
\hline 68 & + & + & + & + & + & + & + & + \\
\hline 70 & + & + & + & - & + & - & + & + \\
\hline 75 & + & + & + & + & + & + & + & + \\
\hline 92 & - & + & + & - & + & + & + & + \\
\hline 116 & + & + & + & + & + & + & + & + \\
\hline 132 & + & + & + & + & + & + & + & + \\
\hline 145 & - & - & + & $\operatorname{tr}$ & $\mathrm{tr}$ & - & + & tr \\
\hline 160 & + & + & + & + & + & + & + & + \\
\hline 175 & - & - & - & + & - & + & - & - \\
\hline
\end{tabular}

$-=$ Absent $; \mathrm{tr}=$ trace $;+=$ strong.

MRSA antiserum, types 1 and 2 were indistinguishable and type 3 showed one difference at mol. wt $145 \times 10^{3}$ from these two types (table II; fig. 3). When the isolates were fingerprinted with the $S$. epidermidis antiserum, there were three differences between types 1 and 2 , and 2 and 3 , and two differences between types 1 and 3 , and 7 and 8 (table III, fig. 4). When fingerprinted with the human antiserum, there were two differences between types 1 and 2, 1 and 3 and only one difference between types 7 and 8 . All other combinations with each antiserum showed at least three differences. When the results of the three antibody probes were combined only the combinations of type 1 versus type 2 (four differences) and type 1 versus type 3 (five differences) produced less than six differences between strains. The combination of type 2 and type 3 produced seven differences. The antibiograms (table V) and phage-typing results (table VI) confirm the similarity between these three types. Type 1 is a rifampicin-resistant variant of the epidemic MRSA (type 2). Type 3 was phage-typed with phage $83 \mathrm{~A}$ but was otherwise a typical epidemic MRSA. The other types all showed considerable differences from the epidemic MRSA in both antibiograms (table $\mathrm{V}$ ) and phage typing (table VI).

\section{Discussion}

This paper described a new technique for fingerprinting MRSA strains. It confirms the finding that the Coomassie blue-stained protein profiles are virtually identical for all isolates within the species (Clink and Pennington, 1987). It demonstrates that there are at least eight different types when the same antigenic extracts are immunoblotted against three different antibody probes.

The system differed from phage typing in that all isolates were typable. Type 8 did not type with any

Table IV. Results of the human antiserum fingerprinting of the eight type strains

\begin{tabular}{|c|c|c|c|c|c|c|c|c|}
\hline \multirow{2}{*}{$\begin{array}{l}\text { Band } \\
\text { Mol. wt } \\
\left(10^{3}\right)\end{array}$} & \multicolumn{8}{|c|}{ Presence of the band in type } \\
\hline & 1 & 2 & 3 & 4 & 5 & 6 & 7 & 8 \\
\hline 38 & + & + & $\mathrm{tr}$ & $\operatorname{tr}$ & $\operatorname{tr}$ & $\operatorname{tr}$ & + & + \\
\hline 40 & + & + & $\operatorname{tr}$ & $\operatorname{tr}$ & tr & $\operatorname{tr}$ & + & + \\
\hline 50 & tr & + & $\operatorname{tr}$ & $\operatorname{tr}$ & $\operatorname{tr}$ & $\operatorname{tr}$ & + & + \\
\hline 54 & $\operatorname{tr}$ & $\operatorname{tr}$ & $\operatorname{tr}$ & + & $\operatorname{tr}$ & $\operatorname{tr}$ & + & + \\
\hline 57 & - & - & - & + & - & - & + & + \\
\hline 61 & + & + & + & + & + & + & + & + \\
\hline 68 & + & + & + & + & + & + & + & + \\
\hline 70 & + & + & + & + & + & + & + & + \\
\hline 75 & + & + & + & - & $\mathrm{tr}$ & $\operatorname{tr}$ & + & + \\
\hline 92 & + & + & + & + & + & + & + & + \\
\hline 116 & + & + & + & + & + & + & + & + \\
\hline 132 & + & + & + & - & $\operatorname{tr}$ & + & - & - \\
\hline 145 & + & - & + & - & + & - & - & - \\
\hline 160 & + & + & + & + & + & + & $\operatorname{tr}$ & - \\
\hline 175 & - & - & - & + & - & + & $\mathrm{tr}$ & $\mathrm{tr}$ \\
\hline
\end{tabular}

$-=$ Absent $; \mathrm{tr}=$ trace $;+=$ strong 
Table V. Antibiograms of the eight types

\begin{tabular}{l|ccccccccccc}
\hline & \multicolumn{10}{c}{ Type } & \multicolumn{10}{c}{ Sensitivity to } \\
\cline { 2 - 13 } & Clin & Ery & Fuc & Gent & Pen & Meth & Chlo & Neo & Rif & Tet & Vanc \\
\hline 1 & R & R & S & R & R & R & S & S & R & R & S \\
2 & R & R & S & R & R & R & S & S & S & R & S \\
3 & R & R & S & R & R & R & S & S & S & R & S \\
4 & R & R & S & S & R & R & S & R & S & R & S \\
5 & R & R & R & S & S & R & S & R & S & R & S \\
6 & R & R & S & S & R & R & R & R & S & R & S \\
7 & R & R & S & R & R & R & S & R & R & R & S \\
8 & R & R & R & R & R & R & R & R & R & R & S \\
\hline
\end{tabular}

$\mathrm{R}=$ resistant; $\mathrm{S}=$ sensitive. Clin, clindamycin; Ery, erythromycin; Fuc, fucidic acid; Gent, gentamicin; Pen, penicillin; Meth, methicillin; Chlo; chloramphenicol; Neo, neomycin; Rif, rifampicin; Tet, tetracycline; Vanc, vancomycin.

Table VI. Results of phage typing

\begin{tabular}{c|ll}
\hline & \multicolumn{2}{|c}{ Phage type at } \\
\cline { 2 - 3 } Type & RTD & RTD $\times 100$ \\
\hline $1^{\text {a }}$ & NT & NT \\
$2^{\text {a }}$ & NT & 85 \\
3 & NT & $83 \mathrm{~A} / 85$ \\
4 & $47 / 54 / 75$ & $29 / 47 / 54 / 75 / 77 / 85$ \\
5 & 75 & 75 \\
6 & $47 / 54$ & $42 \mathrm{E} / 47 / 53 / 54 / 75 / 85$ \\
7 & NT & $6 / 42 \mathrm{E} / 53 / 54 / 75 / 85 / 81$ \\
8 & NT & NT \\
\hline
\end{tabular}

${ }^{a}$ typed with phages $88 \mathrm{~A}$ and 932 in addition. $\mathrm{NT}=$ non-typable.

of the phages tested and type 1 only typed with the experimental phages $88 \mathrm{~A}$ and 932 . Phage typing has the additional problem that there is considerable variation in the results obtained with the epidemic strain. Most type weakly with phage 85 , some strongly with phage 84 and others with experimental phages $88 \mathrm{~A}$ and 932 (Marples et al., 1986). Reproducibility was much better than with a previous immunoblot fingerprinting system developed for C. albicans (Lee et al., 1986), in which it was important to compare all isolates on the same gel. This approach also had to be used with the $\left[{ }^{35}\right.$ S]-methionine labelled typing system for $S$. aureus (Stephenson et al., 1986). Variation in the results obtained with the method of staphylococcal fingerprinting described here was sufficiently small for the direct comparision of immunoblots between gels to be possible, provided that the same batch of antiserum was used for probing.

The discrimination produced by each individual antibody probe can be assessed by its ability to distinguish between two types. It was difficult to separate types 1,2 , and 3 with any of the three antibody probes, but these three types were also very similar on phage typing and antibiograms. The human serum and rabbit $S$. epidermidis antiserum identified only slight differences between types 7 and 8 despite considerable variation in their phage typing (table V), antibiograms, (table VI), and immunoblots probed by the rabbit anti-MRSA antiserum. Apart from these exceptions all of the antibody probes were capable of distinguishing between each of the types.

In terms of the epidemiology and pathogenicity of the MRSA, these results have several consequences. Type 2 represents the epidemic strain which is virulent and capable of spreading to cause major outbreaks. This type included four isolates from Australia confirming the similarity of the isolates from that country to the UK epidemic strain. Types 1 and 3 were very similar. The ability to identify these isolates by their immunoblot profile from other methicillin resistant staphylococci should facilitate documentation of the spread of the epidemic strain.

Type 4 consists of six isolates from Pinderfields Hospital. These fingerprinted as a unique group regardless of which antibody probe was used. They constitute a group of isolates with reduced virulence compared to the outbreak strain (Lacey et al., 1986) and can now be seen to have a totally different fingerprint confirming that they constitute a different micro-organism. Types 5 and 8 are unusual organisms with unique antibiograms (tables I and VI). Types 6 and 7 represent strains from small clusters of cases. They did not cause large outbreaks but could be isolated from several patients in the 
hospitals involved. These clusters were largely self limiting in contrast to the large outbreak caused by the EMRSA.

In summary, this paper demonstrates the use of immunoblotting to identify isolates of the EMRSA and differentiate them from other methicillin resistant strains. These strains, despite being

\section{REFERENCES}

Boyce J M, Landry M, Deetz T R, DuPont H L 1981 Epidemiological studies of an outbreak of nosocomial methicillin-resistant Staphylococcus aureus infections. Infection Control 2: 110-116.

Bradley J M, Noone O, Townsend D E, Grubb W B 1985 Methicillin-resistant Staphylococcus aureus in a London hospital. Lancet 1 : 1493-5.

Burnie J P, Matthews R C 1987 Immunoblot analysis: a new method for fingerprinting hospital pathogens. Journal of Immunological Methods $100: 41-46$.

Clink J, Pennington T H 1987 Staphylococcal whole-cell polypeptide analysis : evaluation as a taxonomic and typing tool. Journal of Medical Microbiology 23:41-44.

Combined working party of the Hospital Infection Society and British Society for Antimicrobial Chemotherapy 1986 Guidelines for the control of epidemic methicillin resistant Staphylococcus aureus. Journal of Hospital Infection 7:193201.

Crossley K, Landesman B, Zaske D 1979 An outbreak of infections caused by strains of Staphylococcus aureus resistant to methicillin and aminoglycosides. II Epidemiological studies. Journal of Infectious Diseases 139: 280-287.

Hone R et al. 1981 Bacteraemia in Dublin due to gentamicinresistant Staphylococcus aureus. Journal of Hospital Infection 2:119-26.

Krikler S J, Pennington T H, Petrie D 1986 Typing of strains of Staphylococcus aureus by Western Blot analysis of culture supernates. Journal of Medical Microbiology 21:169-171.

Lacey R W, Barr K W, Barr V E, Inglis T J 1986 Properties of methicillin resistant Staphylococcus aureus colonising patients in a burns unit. Journal of Hospital Infection 7:13748. methicillin resistant, are often less virulent and less likely to cause major epidemics so that their distinction from the EMRSA is of considerable importance.

We thank Dr R. Marples for phage typing, Dr G. Duckworth for the isolates in types 3 and 5, Professor R. Lacey for the isolates in type 4 and Dr R. Peel for the isolates in types 6 and 7.

Lacey R W, Chopra I 1975 The effect of plasmid carriage on the virulence of Staphylococcus aureus. Journal of Medical Microbiology 8: 137-147.

Laemmli U K 1970 Cleavage of structural proteins during the assembly of the head of bacteriophage T4. Nature 227:680685 .

Lee W, Burnie J P, Matthews R C 1986 Fingerprinting Candida albicans. Journal of Immunological Methods 93:177-182.

Marples R R, Richardson J F, de Saxe M J 1986 Bacteriological characters of strains of Staphylococcus aureus submitted to a reference laboratory related to methicillin resistance. Journal of Hygiene 96 : 217-223.

McDonald M, Hurse A, Sim K N 1981 Methicillin-resistant Staphylococcus aureus bacteraemia. Australian Medical Journal 2: 191-194.

Parker M T 1972 Phage-typing of Staphylococcus aureus. In: Norris J R, Ribbons D W (eds) Methods in Microbiology. 7B. London Academic Press, pp 1-28.

Peacock J E Jr, Moorman D R, Wenzel R P, Mandell G L 1981 Methicillin-resistant Staphylococcus aureus: microbiological characteristics, antimicrobial susceptibilities and assessment of virulence of an epidemic strain. Journal of Infectious Diseases 144: 575-582.

Sanderson P J 1986 Staying one jump ahead of resistant Staphylococcus aureus. British Medical Journal 293:573574.

Shanson D C, Johnstone D, Midgley J 1985 Control of a hospital outbreak of methicillin-resistance Staphylococcus aureus infection: value of an isolation unit. Journal of Hospital Infection 6:285-292.

Stephenson J R, Crook S J, Tabaqchali S 1986 New method for typing Staphylococcus aureus resistant to methicillin based on sulphur-35 methionine labelled proteins: its application in an outbreak. British Medical Journal 293:581-583. 